\title{
UNUSUAL EXTRA-ARTICULAR MANIFESTATION OF RHEUMATOID ARTHRITIS: PNEUMOMEDIASTINUM
}

\author{
Tatiana Melo Fernandes ${ }^{1, \star}$, Mariana D’Oliveira Bulhões da Costa ${ }^{1}$, Franciele Cristina Ferreira Pereira ${ }^{1}$, Walter Carlos de Oliveira \\ Bohrer $^{1}$, Bartira Souza Melo ${ }^{1}$, Carlos de Oliveira Nascimento ${ }^{1}$, Maressa Barbosa Beloni Lirio ${ }^{1}$
}

1.Hospital Naval Marcílio Dias, Rio de Janeiro (RJ), Brazil.

*Corresponding author: tatianamelofernandes@gmail.com

\section{BACKGROUND}

Rheumatoid arthritis (RA) is a systemic autoimmune disease that can manifest in different ways. Although symmetrical polyarthritis of small joints of the hands and wrists is ordinary in the disease, it is important to know the atypical forms of presentation. Pulmonary involvement, manifested as interstitial lung disease (ILD), is not a rare condition, but it is uncommon at the time of diagnosis. Structural changes in ILD can lead to the formation of spontaneous pneumomediastinum, an extremely rare condition associated with RA and not well described in the medical literature.

\section{CASE REPORT}

A 47-year-old female patient had bariatric surgery previously, started dyspnea on mild exertion and a drop in saturation by pulse oximetry associated with symmetric inflammatory polyarthritis in hands, feet, knees and ankles. A chest computed tomography (CT) scan showed ground-glass, fibrosis, bilateral and diffuse honey-combing, especially in the periphery and lower lobes, in addition to extensive pneumomediastinum. Laboratory tests showed anemia, normal leukocytes, high inflammatory tests and positives rheumatoid factor, antinuclear factor and anti-citrulline peptide. Blood and urine cultures were performed to rule out infectious causes. The pneumomediastinum has treated with conservative measures without response. Thoracic surgery team has not recommended surgical approach due to hemodynamic stability. Transthoracic echocardiogram shows minor pericardial effusion and pulmonary artery pressure at upper limit. Radiograph of hands and wrists were normal. The team has not found signs of esophageal laceration through upper digestive endoscopy. Treatment was started with pulse therapy with methylprednisolone $1 \mathrm{~g}$ for three consecutive days associated with cyclophosphamide $1 \mathrm{~g}$ monthly for six months. She was discharged from the hospital with oxygen support and started using nintedanib $150 \mathrm{mg}$ twice a day orally. She had a slight improvement in dyspnea and a reduction of supplemental oxygen need. A new chest image after three months showed a significant reduction in pneumomediastinum, but aggravate pulmonary fibrosis, and it was decided to start rituximab $1 \mathrm{~g}$ in two infusions.

\section{CONCLUSION}

Pneumomediastinum can occur spontaneously or due to a traumatic cause and can be related to rheumatic diseases, especially dermatomyositis. It presents with chest pain, dyspnea, dry cough, dysphonia, vertigo and subcutaneous emphysema. Less frequently there could be pain and discomfort in the oropharynx, dysphagia and odynophagia. Diagnosis is made by imaging tests, mainly chest $\mathrm{CT}$. It is self-limiting, but the use of supplemental oxygen for gas reabsorption and immunosuppression may be necessary to treat its underlying etiology.

\section{KEYWORDS}

Rheumatoid arthritis, Pneumomediastinum, Unusual extra-articular manifestation. 